\title{
Dependence of Coefficient of volumetric thermal expansion (CVTE) of glass fiber reinforced (GFR) polymers on the glass fiber content
}

\author{
V. La Carrubba ${ }^{(a)}$ (ख), M. Bulters ${ }^{(b)}$, W. Zoetelief ${ }^{(b)}$ \\ (a) Chemical Engineering Department, University of Palermo - Viale delle Scienze, 90128 \\ Palermo, Italy \\ (b) DSM Research - P.O. Box 18,6160 MD, Geleen. The Netherlands \\ E-mail: Iacarnbba@dicpm.unipait; Fax: +39-091-6567280
}

Received: 15 Decemher 2006 / Revised version: 19 July 2007 / Accepted: 12 August 2007

Published online: 25 August 2007 - 10 Springer-Verlag 2007

\section{Summary}

In a Glass Fiber Reinforced (GFR) polymer, the coefficient of volumetric thermal expansion CVTE (determined as a sum of the coefficients of linear thermal expansion CLTE's for the three principal directions) is sometimes much smaller than the value predictable on the basis of well acquainted models. such as Chow model. taking into account fibers anisotropy and aspect ratio.

A detailed investigation of the CVTE of unfilled and GFR thermoplastics (polyethyleneterephthalate PET, polybuthyleneterephthalate PBT, polyamide 6 PAK, polyamide 4,6 PA46, polycarbonate PC) was performed throngh Pressure-Volume Temperature (PVT) measurements. In particular, it was found that CVTE is always much lower than the zeroth order "expected value", defined according to the "rule of mixture". The aspect ratio plays a major role, since in the case of polymers filled with glass spheres the rules of mixtures applies for the resulting CVTE. Finally, the nature of the matrix is of paramount importance: a GFR polymer with an amorphous matrix (PC) strictly follows the rule of mixture for CLVE even for highly anisotropic fillers cxhibiting large aspect ratios (20 to 30 ).

\section{Introduction and background}

A major issue for polymers in engineering applications is to reduce the thermal expansion coefficient so as to achieve dimensional stability more comparable in metals. Numerous studies have examined how filler shape, size, and volume fraction influence the thermal expansion of polymer composites. Long fiber composites have a significantly lower linear thermal expansion cocfficient than the matrix polymer [1]. Anisotropic thermal ixpansion bebavior for injection-molded specimens has been observed, the expansion coefficient in the flow direction (FD) being lower than in the perpendicular direction $[2,3]$.

Filler geometry can also greally affect physical proper-ties of composites; e.g. high aspect ratios normally contribute to greater reduction in thermal expansion [4]. 
Enhancement of dimensional stability is expected when a filler with high modulus and low thermal expansion coefficien! is dispersed in a matrix of lower modulus and higher thermal expansion coefficient owing to simple mechanical restraints.

Last but not least, an accurate knowledge and prediction of thermal expansion coefficients (hence shrinkage) across different directions in both unfilled and glass filled injection molded samples is a crucial point for an effective prevention of warpage occumence, often due to asymmetric shrinkage and/or shrinkage differences in differen directions (orientation shrinkage) [5].

The linear thermal expansion coefficient along a direction $/$ is dcfincd as:

$$
\alpha_{i}=1 / l \times \partial l / \partial T=\partial \ln (l) / \partial T
$$

being $l$ sample length. The volumetric thermal expansion coefficient is defined as:

$$
\alpha_{v}=1 / v \times \partial v / \partial T=\partial \ln (v) / \partial T
$$

where $v$ is the specific volume.

In a homogeneous material the relationship between the two coefficients of thermal expansion is such that $\alpha_{v}=\alpha_{x}+\alpha_{y}+\alpha_{z}$, where $x, y$ and $z$ are three mutually orthogonal directions; obvinusly in case of an isotropic material $\alpha_{y}=3 \alpha_{1}$.

In a Glass Fiber Reinforced (GFR) polymer, the thermal expansion coefficient may be expected to result from the rule of mixtures; the contribution of the thermal expansion of the polymer matrix $\left(\alpha_{0}\right)$ and the filler $\left(\alpha_{g}\right)$, are weighed by their respective volumetric fractions $f[6]$ :

$$
\alpha_{\text {OFR }}=\alpha_{p} f_{P}+\alpha_{R}\left(1-f_{r^{\prime}}\right)
$$

More refined models, such as the Chow model [7], or the Schapery model [8] take into account the filler anisotropy and aspect ratio (through the deformation state).

The presence of large aspect ratio flllers, like fibers, induces mechanical constraints on the polymer matrix resulting in a lower "effective" coefficient of volumctric thermal expansion $\alpha_{v}$, depending on several factors: the nature of the polymer matrix, the fiber distribution and hence the inter-fiber distance, and the matrix/fiber adhesion. Recent work on Polyamide 6 nanocomposites [9] has shown that the $\alpha_{V}$ of these systems (determined as a sum of the Coefficients of Lincar Thermal Expansion in the three principal directions) is sometimes much smalier than the value predicted by these models. However, the use of more accurate closure approximations applied to the Tucker-Liang [10] and Mori-Tanaka [11-12] model have notably improved the cvaluation of thermoelastic properties of injection molded short-fiber composites [13]. The purposc of the present experimental work was to determine and compare the $\alpha_{V}$ of unfilled (UF) and GFR crystalline (poly-ethylene-terephthalate PET, polybuthylene-terephthalate PBT, polyamide-6 PA6, polyamide-4,6 PA46) and amorphous thermoplastics (polycarbonate PC), in order to validate the proposed models [7-9] and to highlight the dependence of this coefficient upon the volumetric filler content. Furthermore the influence of fiber aspect ratio and of the nature of the matrix (amorphous vs. semicrystalline) is presented and shortly discussed.

\section{Experimental}

Volumetric coefficients of Expansion were determined through Pressure-Volume Temperature (PVT) analysis. The apparatus used was the "Gnomix PVT", based on 
the use of a confining fluid and a special "sample cup", enstring a hydrostatic state of stress (i.e. a true pressure) in the sample at all times and phases [14].

Linear thermal expansion coefficients across directions perpendicular and parallel to flow were measured by moans of a standard apparatus according to ASTM E831.

Rectangular plates $800 \mathrm{~mm}$ long, $500 \mathrm{~mm}$ wide and $1.5 \mathrm{~mm}$ thick were injection moulded in a mould plate with a sprue gate positioned in the centre (see fig. 1) by using standard injection moulding conditions. Macrials and compositions as reported in table 1 . Standard short glass fibers approximately $300 \mathrm{Hm}$ long with an $\mathrm{L} / \mathrm{D}$ ranging from 10 to 15 where compounded in different proportions with different polymeric matrices, including both semicrystalline (PET, PBT, PA46 and PA6) and amorphous polymers (PC). In one case (PA6) glass beads with a diumeter of approximately $30 \mu \mathrm{m}$ were compounded (sec Table 1).

Three injection molded samples per each material composition were taken for the experimental investigations (PVT and Linear Expansion runs).

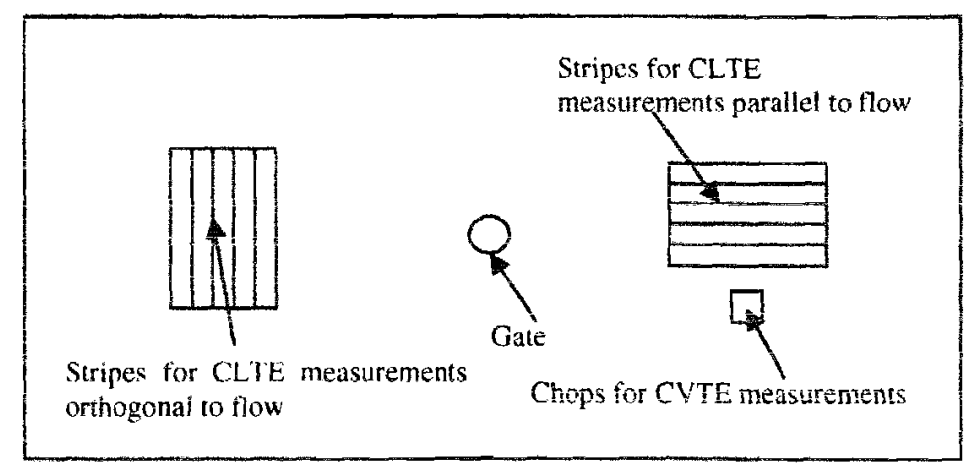

Figure 1: Schematic of the mould plate geometry, indicating samples used for CLTE and CVTE

Table 1: Matcrials analyzed

\begin{tabular}{ll}
\hline Material & Glass fiber content, wt $\%$ \\
\hline PET & 0,50 \\
PBT & 0,35 \\
PA46 & 0,60 \\
PA6 & $0,50,50$ (glass beads) \\
PC & 0.30 \\
\hline
\end{tabular}

For the linear thermal expanvion measurements 5 rectangular stripes $100 \mathrm{~mm}$ iong and $20 \mathrm{~mm}$ wide were cur across directions perpendicular and parallel to flow, as schematically shown in fig. 1 . The stripe length as a function of time was motitored by means of a LINSEIS 8 push-rod Dilatometer type L75/120-LT from 120 to $-15^{\circ} \mathrm{C}$; 5 samples wore moasurcd per material. Typical examples of the raw data, showing averaged $\Delta L$ as a function of temperature for PA46-UF samples cut across parallel and perpendicular lirection are reported in fig. 2 al and b respectively.

On the other hand, samples of 1.2 to $1.5 \mathrm{~g}$ werc taken from the plate zones with fully developed flow (hence reasonably characterized by the largest fiber orientations), i.e. in betwecn the gate and the plate edges but sufficiently far from both (see fig. 1), in order to investigate the depcndicnci of $\alpha_{V}$ upon the nature of the matrix, the presence 

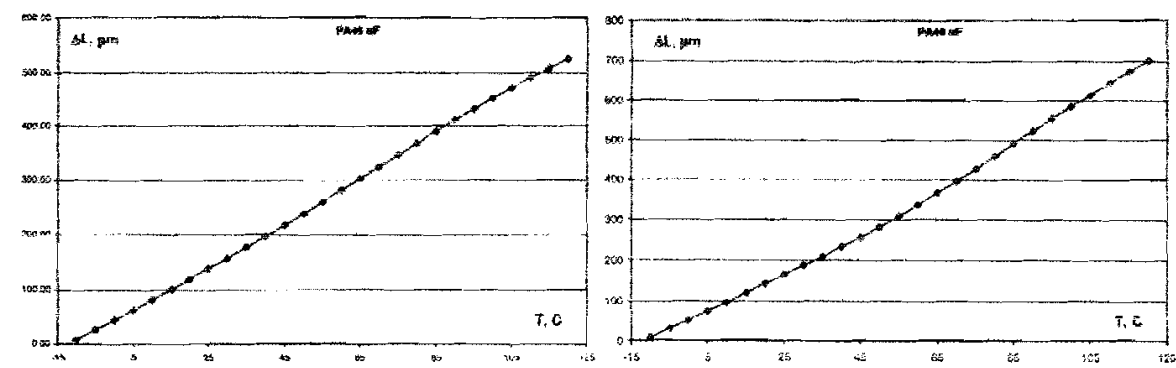

Figure 2: $\Delta \mathrm{L}$ vs. temperature as recorded by the apparatus for measuring $\alpha_{l}$. a (lcft): PA46-uF parallel direction. $\mathrm{B}$ (right); $\mathrm{PA} 46-\mathrm{uF}$ perjendicular direction)

of glass fibers, and their aspect ratio. However, the orientation distribution across the plate thickness was nol examined, going beyond the scope of the present investigation, aiming to provide easy-to-handle comrelations among the thermal expansion behavior and the volumetric fiber content in short fiber polymer composites.

These samples were subjected to successive isothermal compressions from 30 to $60^{\circ} \mathrm{C}$, at subsequent pressures ranging from 10 to $200 \mathrm{MPa}$ at intcrvals of $10 \mathrm{MP}$. From these data $\alpha_{v}$ was calculated. As an example, fig. 3.a shows the dependence of specific volume upon temperature in the range from 30 to $60^{\circ} \mathrm{C}$ for the PBT GFR sample. it is opportune to recail that the curve at ambient pressure is automatically provided by the software of the PVT instrument via extrapolation of higher pressure data [14]. The thermal expansion coefficient is determined from the slope of the plot of $\ln (v)$ versus temperature, see eq (2), as schematically shown in fig. 3.6 for a PBTGFR and a PA6 uF at different pressure values.

Finite Element (FE) simulations of the stress state on a single glass fiber surrounded by a polymer matrix (volumetric fiber content of $25 \%$ ) were carried out with the help of the MSC-MARC software. A pure isotropic elastic behavior was assumed as a first order approximation with periodic boundary conditions (for the displacements), and

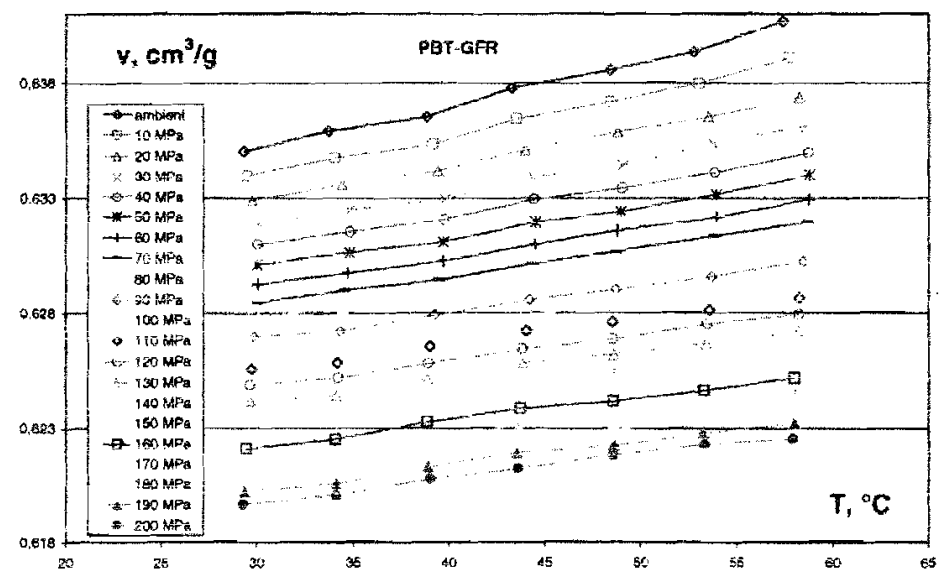

Figure 3.a: Specific volumc vs. T for different pressures 

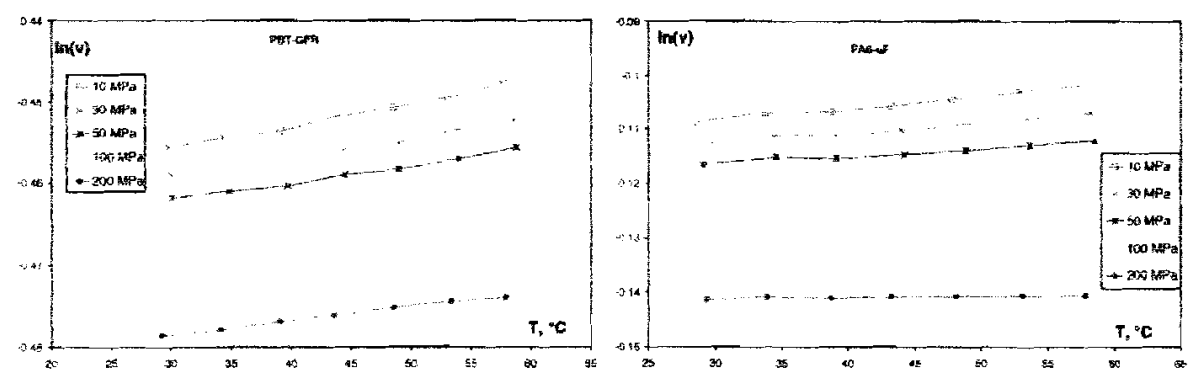

Figure 3.b: Log of specific volume vs. T for different pressures. Left: PBT-GFR; right: PA6 uF

a cooling step of $50^{\circ} \mathrm{C}$ ("shrinkage load") down to room temperature was examined, reporting the results in terms of equivalent von Mises' iso-stress curves (being the frozen stresses generated by the thermal contraction owing to cooling).

\section{Results and discussion}

For all the materials investigated in this (narrow) temperature range, $\alpha_{V}$ turns out to be constant with temperature, and decreases with pressure, see figures 4 to 8 .

Results for the unfilled (UF) PBT and the PBT/GFR are presented in fig. 4, which shows that $\alpha_{V}$ is strongly reduced by the presence of the glass fibers. The actual value of $\alpha_{v}$, over the whole pressure range, of the glass filled samples is some 25-30\% lower than calculated through eq (3), i.e. by a mere volumetric weighed average of the $\alpha_{V}$ 's of the pure polymer and of the glass (see triangles in fig. 4). The volumetric content was calculated based on the average matrix densily (measured in the PVT at different pressures), on the glass fiber density and on the particular weight fraction. The compressibility of glass may be found in literature [15], its value being definitely negligible with respect to the typical compressibility of a polymer matrix in the explored pressure range (maximum pressure $200 \mathrm{MPa}$ ). The decrease of $\alpha_{v}$ with respect to the "rule of mixtures" value predicted by several models (see for instance the Chow model) is less than $10 \%$ for $25 \%$ glass fiber volumetric content and a fiber aspect ratio of 10 [7]. As a matter of fact, according to the Chow model, the thermal expansion coefficient of a GFR polymer may be calculated by:

$$
a_{t i r R}=\alpha_{p}+\left(k_{g} / k_{m}\right) \times\left(\alpha_{g}-\alpha_{p}\right)\left(G_{1}+2 G_{3}\right) \phi /\left(2 K_{1} G_{3}+G_{1} K_{3}\right)
$$

where $k_{i}$ are the bulk moduli, $\phi$ is the volumetric fiber content and $K_{i}, G_{i}$ are coefficients depending on the Poisson's ratio of the polymer and on the aspect ratio of the glass. It may be easily shown [7] that eq (4) does not supply values significantly deviating from the values given by eq (3): this is visibly shown in fig. 4 , where the predictions of the volumetric thermal expansion according to the Chow Model are reported, almost coinciding with the values provided by the rule of mixtures.

The deviation between measured and expected values (based on the rule of mixtures) is cven more pronounced in the case of the PA46 and PA46-GFR, as shown in fig. 5. A slightiy different behavior is exhibited by PET-based systems (fig. 6), where the $\alpha_{V}$ of PET-GFR is much less dependent on pressure. It is also worthwhile noticing that the absolute value of $\alpha_{v}$ 's of the PET-based systems (ranging from $1.2 \mathrm{e}$ to $2.3^{*} 10^{-4}$ ) is much lower than the one of the PBT-based systems (from 1.7 to $3.5^{*} 10^{-4}$ ). 


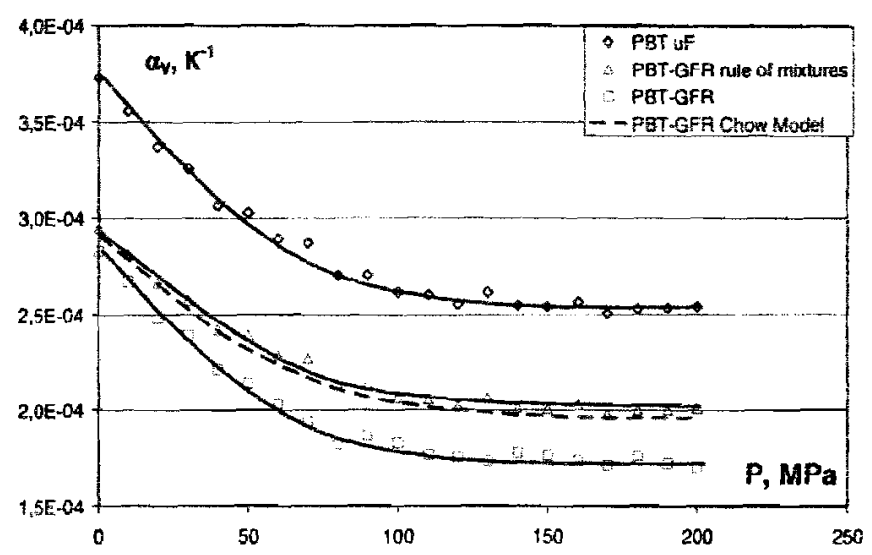

Figure 4: $\alpha_{V}$ for PBT: UF, GFR, eq (2) and Chow Model (eq 4)

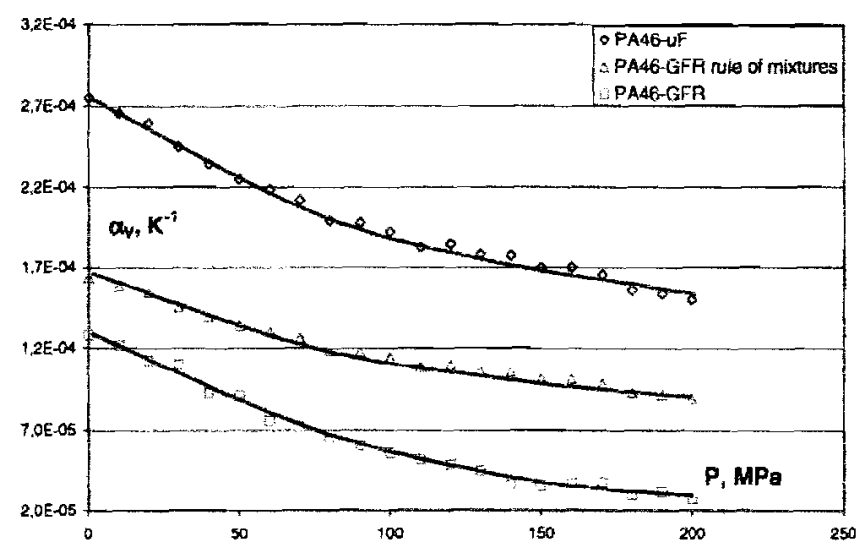

Figure 5: $\alpha_{V}$ for PA46: UF, GFR and eq (2)

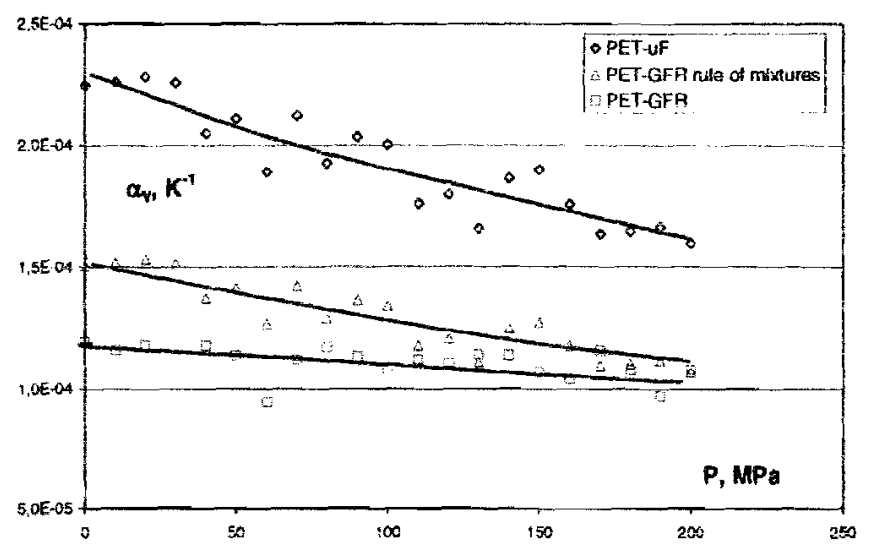

Figure 6: $\alpha_{v}$ for PET: UF, GFR and eq (2) 


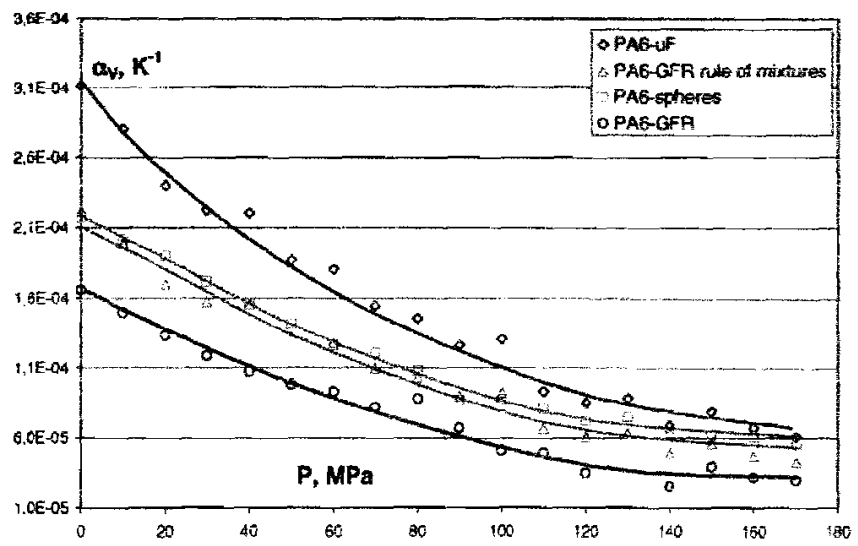

Figure 7: $\alpha_{V}$ for PA6: UF, GFR, spheres and eq (2)

That the aspect ratio of the filler affects the expansivity is illustrated in fig. 7, where $\alpha_{v}$ 's for UF PA6 and for PA6 filled with equal volumetric content of glass fibers and glass beads (aspect ratio equal to one), respectively, are compared.

While in the case of GFR PA6 the behavior is in line with the one exhibited by the other systems whose matrix is a semicrystalline polymer, in the case of PAo filled with glass spheres the measured values of $\alpha_{V}$ almost coincide with the one predicted by the rule of mixtures (eq.3). An increase of the aspect ratio from 1 (spheres) to about 30 (the typical aspect ratio of the fibers used in glass fiber filled systems) corresponds to a relative decrease in the thermal expansion coefficient of about $25 \%$.

Finally, $\alpha_{V}$ as a function of pressure for the PC-UF and the PC-GFR are reported in fig. 8. Like in the case of PA6 filled with glass spheres, the measured values of the thermal expansion coefficient do follow the rule of mixtures over the whole pressure range, showing that also the matrix properties are relevant.

Another interesting heuristic conclusion can be drawn by defining the "excess normalized coefficient of thermal expansion" $\alpha_{e, n}$ as follows:

$$
\Gamma_{e, n}=\left(\alpha_{r a m}-\alpha_{\text {mess }}\right) / \alpha_{\text {rom }}
$$

expressing the deviation of the experimentally measured thermal expansion $\alpha_{\text {meas }}$ from the "reference value", $\alpha_{r m}$, which is the expected value of expansivity calculated using the rule of mixtures (eq.3). Although both $\alpha_{\text {meas }}$ and $\alpha_{\text {rom }}$ exhibit a significant dependence upon pressure (at least up to $100 \mathrm{MPa}$ ), however their difference (normalized by $\alpha_{\text {rom }}$ ) shows only a mild dependence upon pressure, therefore the average value of $\Gamma_{e, n}$ in the range $0-50 \mathrm{MPa}$ (the typical span of pressure values normally employed in polymer processing) will be reported in the following for each material.

This parameter indicates how large the departure of $\alpha_{\mathrm{V}}$ is from "ideality" (the rule of mixtures). Fig. 9 reports $\Gamma_{e, n}$ as a function of the volumetric fiber content.

It should be noticed that all points align quite well on a straight line passing through the origin, indicating the existence of a correlation between the volumetric fiber glass content and the decrease of $\alpha_{V}$ with respect to the "expected" value. It is worth stressing that the data refer to 4 different materials (PET, PBT, PA6 and PA46). 


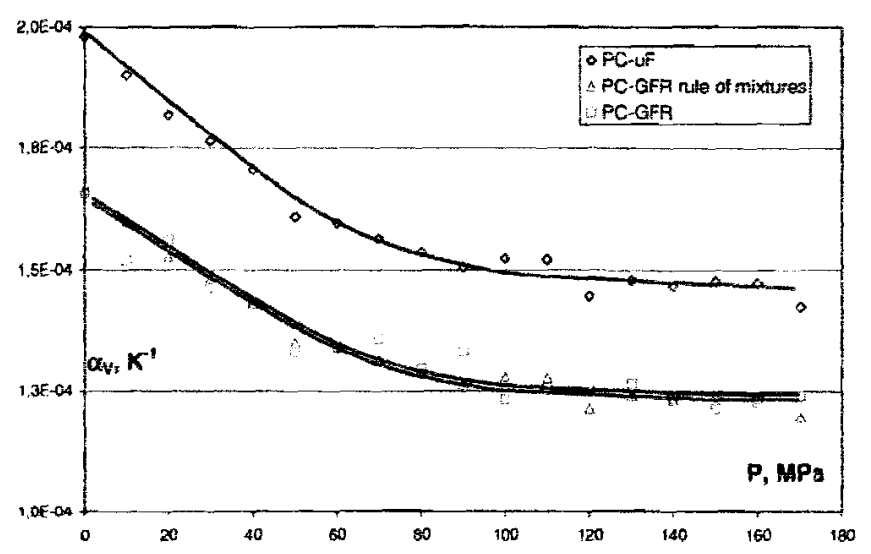

Figure 8: $\alpha_{\mathrm{F}}$ for PC: UF, GFR and eq (2)

In other words, the presence of glass fibers exerts a significant constraint on the surrounding polymeric material, hindering its expansion and hence lowering its $\alpha_{v}$. This constraining action is almost independent of the nature of the matrix (if semicrystalline), at least for the polymers studied in this experimental campaign.

Furthermore, the results of fig. 9 may provide some quantitative information about this "constraining effect". As a matter of fact, the slope of the curve is equal to 0.6 , which means that the presence of fibers determines a further decrease of the volumetric thermal expansion coefficient with respect to the one determined on the basis of a volumctric additivity, see eq (3), this decrease being equivalent to an "effective" glass fiber content approximately equal to a $60 \%$ increase with respect to the real content. More exactly, since

$$
\left(\alpha_{\text {rom }}-\alpha_{\text {treas }}\right) / \alpha_{\text {ram }}=0.6 \times f_{s}
$$

where $f_{g}$ is the volumetric fiber fraction, and since

$$
\alpha_{\text {rom }}=\alpha_{p} \times\left(1-f_{g}\right)+\alpha_{g} \times f_{g}
$$

one can calculate an "equivalent" volumetric glass fiber fraction $f_{p}^{*}$, defined as:

$$
\alpha_{\text {mens }}=\alpha_{p} \times\left(1-f_{g}^{*}\right)+\alpha_{g} \times f_{g}^{*}
$$

or, equivalently:

$$
f_{g}^{*}=\left(\alpha_{p}-\alpha_{\text {imeas }}\right) /\left(\alpha_{p}-\alpha_{g}\right)
$$

From eqs (6) (7) and (9) the relalionship between $f_{g}^{*}$ and $f_{g}$ can be found:

$$
f_{g}^{*} / f_{g}=\left(1+0.6 f_{g}\right)+0.6 \alpha_{p} /\left(\alpha_{p}-\alpha_{g}\right)
$$

Since for all polymers it is casy to notice that $\alpha_{p} \gg \alpha_{g}$, one my write with a good approximation:

$$
f_{g}^{*} / f_{g}=1.6+0.6 \times f_{g}
$$




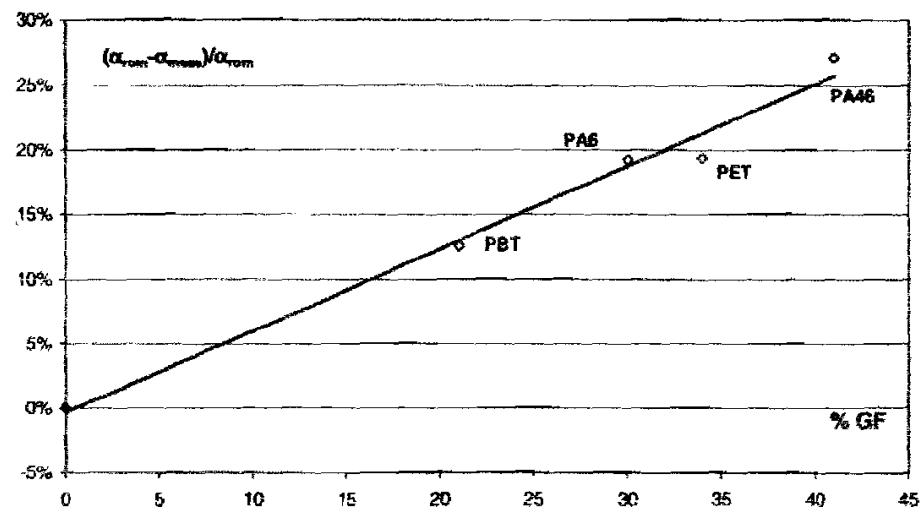

Figure 9: Excess normalized $\alpha_{V}$ vs. vol. fiber content

Therefore, if one wants to determine the volumetric thermal expansion coefficient, the real glass fiber content must be multiplied by a factor reported in eq. 10 . In volumetric terms this means that a portion of polymer around the fiber larger than $60 \%$ of the real fiber volume is hindered in the expansion, with the ratio between the "effective" fiber diameter $D_{e f f}$ and the real fiber diameter $D$ equal to:

$$
D_{e, f} / D \approx \sqrt{1.6+0.6 \times f_{g}}
$$

In order to verify the reasonability of the hypothesis of an "effective" fiber diameter resulting from a compressive state around the fiber, Finite Element (FE) simulations of the stress state on a single fiber surrounded by a PET matrix were carried out (by using the program MSC-MARC). A couling step of $50^{\circ} \mathrm{C}$ ("shrinkage load") in the solid state (down to room temperature) was simulated and the resulting stress state around glass fibers (in terms of equivalent von Mises' stresses) is reported in fig. 10 , which shows that an "effective fiber diameter" exists (larger than the geometric one), whose extent depends upon the definition of a "critical von Mises' stress" above which an effective constraining effect takes place (around $10 \%$ more of the geometrical diameter for a critical value of $100 \mathrm{MPa}$ and around $30 \%$ for a critical value of $70 \mathrm{MPa}$ ).

In other words, a "stress-confined" region around a glass fiber exists, where the volumetric expansion is hindered owing the frozen-in thermal stresses, the final outcome being a net reduction in the volumetric coefficient of thermal expansion, which turns out to be lower than the "expected" value, evaluated according to the rule of mixtures (eq 3).

Similar considcration could be made by looking at the first invariant of the stress tensor, whose contour maps are not reported here for the sake of brevity.

On the other hand, when the matrix is amorphous (PC, see fig. 8) or the fiber aspect ratio tends to 1 (PA6 filled with glass spheres, see fig. 7), the excess normalized $\alpha_{v}$ is always zero, regardless the glass fiber content. The experimental evidence concerning GFR polymers (PC) however deserves further investigations.

In particular, the larger "eflective" fiber diameter (experimentally determined) noticeable in various type of semicrystalline polymers (polyesters and polyamides), suggests the hypothesis of a possible additional contribution of the nucleating effect exerted by the glass fiber onto the surrounding semicrystalline polymer matrix (and 


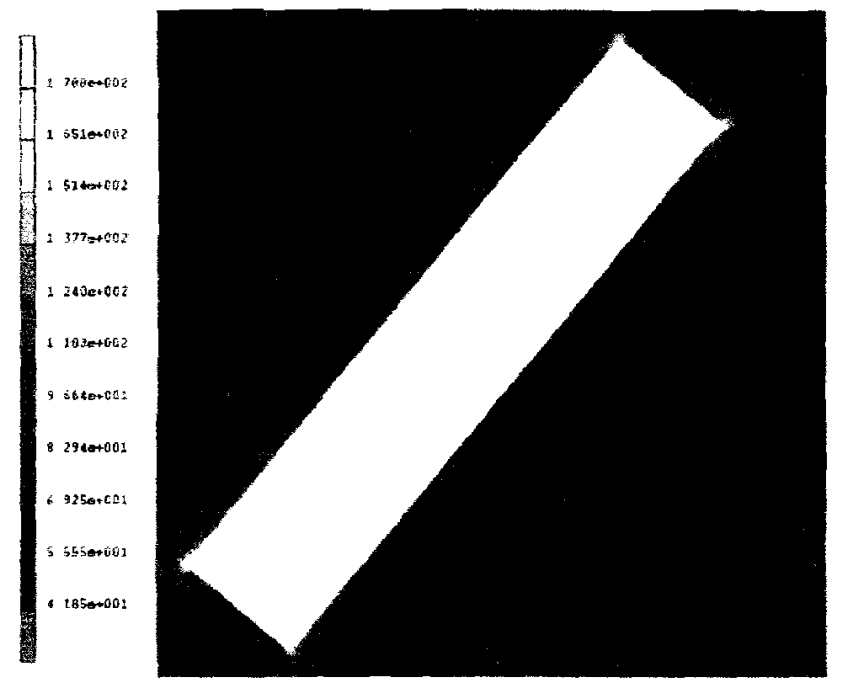

Figure 10: FE equivalent von Mises' Stress for volumetric expansion of a PET matrix around a single glass tiber. Matrix properties: $\mathrm{E}=3.5 \mathrm{GPa}, v=0.394, \mathrm{CLTE}=2.5 * 10^{-4}[\mathrm{l} / \mathrm{K}]$ : fiber properties: $\mathrm{E}=70 \mathrm{GPa}, \mathrm{v}=0.18, \mathrm{CLTE} \approx 0[1 / \mathrm{K}]$

not onto an amorphous matrix). Although this argument sounds reasonable, since it has been frequently observed in polyolefinic matrices (iPP), so-far the experimental evidences concerning polyesters are not supporting it [16].

In any case, the experimental evidence corroborates the hypothesis that the reduction of the thermal expansion coefficient is related to the interplay between the constraining effect induced by the stress state and the specific polymer morphology around the glass fiber.

Coefficients of linear expansion $\alpha_{r}$ across directions perpendicular and parallel to flow were measured by taking the slope of $\ln (\mathrm{L})$ vs. T curve (see eq.1). By ploting $\alpha_{i}$ versus $\alpha_{v}$ for the all the unfilled materials one gets the data reported in fig. 11, $\alpha_{l}$ across parallel and perpendicular direction were measured by the linear thermal

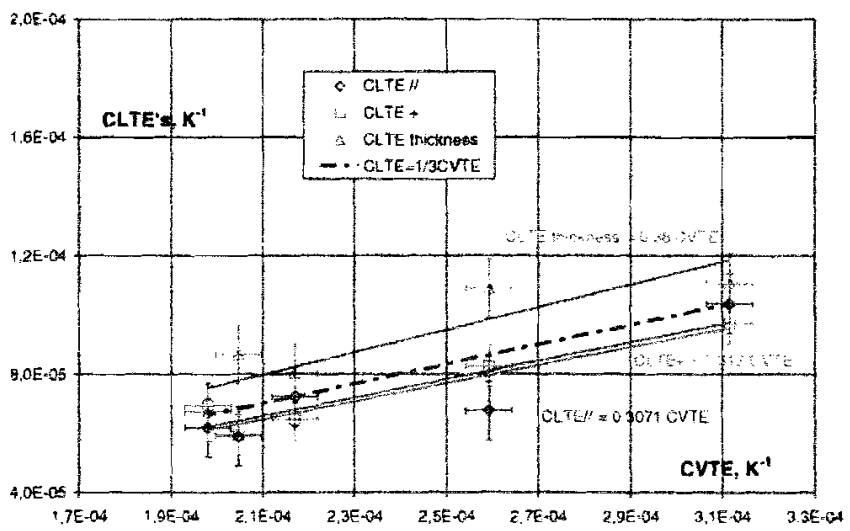

Figure 11: CLTE's versus CVTE for unfilled materiais 


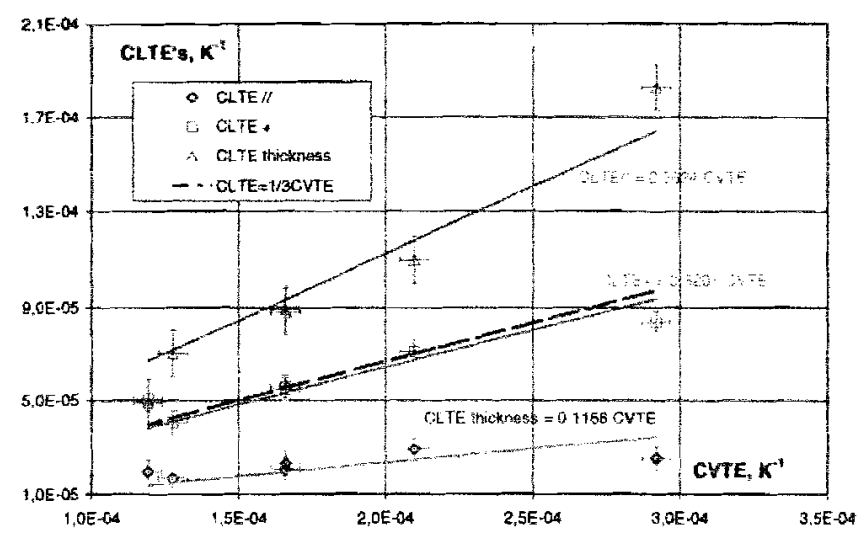

Figure 12: CLTE's versus CVTE for GFR materials

expansion coefficient apparatus, $\alpha_{V}$ values come from the PVT data at ambient pressure and $\alpha_{l}$ across the thickness direction is obtained by subtracting from $\alpha_{V}$ the value of $\alpha_{l}$ parallel and $\alpha_{t}$ perpendicular. It is easy to notice that the threc coefficients do not significantly differ from each other; additionally, the slope of all the curves is very close to $1 / 3$, rigorously valid for isotropic materials. The final conclusion is that for unfilled materials there is not a significant thermal anisotropy.

When iterating the same procedure for glass fiber reinforced materials, the data reported in fig. 12 are obtained. In this case a large thermo-mechanical anisotropy should be noticed, i.e. both an "intrinsic" anisotropy in thermal properties and an induced" anisotropy due to the thermal history experienced during processing. As a consequence of that the coefficient along parallel direction turns out to be very small, the one across perpendicular direction is close to $1 / 3$ and the one across thickness direction is about $2 / 3$.

\section{Conclusions}

The large set of obtained experimental data concerning the coefficients of thermal expansion (volumetric and linear) leads to the following main conclusions:

- the experimentally determined value of volumetric thermal expansion for semicrystalline polymers (polyamides and polyesters) filled with glass fibers is always lower than the "expected value" based on the "rule of mixtures";

- a synthetic parameter, here named "excess normalized coefficient of thermal expansion" $\left(\Gamma_{e, n}\right)$, expressing the deviation of the expcrimentally measured thermal expansion from the "rule-of-mixtures based" expansivity can be suitably applied to describe the observed reduction of the volumetric thermal expansion coefficient;

- in particular, $\alpha_{e, n}$ linearly depends on the glass fiber volumetric content for semicrystalline polymers, whereas one amorphous polymer here tested. PC, behaves differently, since the rule of mixture always applies; this evidence, if confirmed for other composites based on amorphous polymeric matrices, may suggests an interplay between the constraining effect induced by the stress state and the specific polymer morphology around the glass fiber (transcrystalline regions in the vicinity of the fiber surface); further experiments should be however carried out to confirm the hypothesis; 
the filler aspect ratio dominates this behavior, since in semicrystalline polymers) filled with beads $(\mathrm{L} / \mathrm{D}=1)$ the mixture rules applies;

- in injection molded specimens the coefficients of linear thermal expansion are equal to $1 / 3$ of the volumetric ones for unfilled polymers where no thermal anisotropy can be noriced; whereas for GFR materials, a very large thermo-mechanical anisotrony is observed the coefficients of thermal expansion across the thickness directions being much larger than the one across perpendicular direction; finally the coefficient of thermal expansion along the flow direction is about $10 \%$ of the volumetric one, in line with previously reported resuits $[2,3]$.

\section{References}

1. Segal R (1979) Pol Eng Sci $19: 365$

2. Okada A, Kawasumi M, Usuki A (1990) Mater Res Soc Symp Proe 171:45

3. Okuda A. Ustki A (1995) Mater Sci Eng C3:109

4. Lusis 1. Woodhams BT, Xanthos M (1073) Polym Eng Sci 13:139

5. Zeng R. Kernedy P. Phan-Thion N, Fan X-1 J. (1999) Non-Newt Fluid Moch 84:159

6. Papathanasiou TD. Guell DC (1997) Flow-induced alignment in composite materials. Wondhead Publishing limited. Cambridge

7. Chow TS (1978) J Pol Sei Pol Phys 16.967

8. Schapery RA (1968) J Compos Mat 2:380

9. Yoon PJ. Fornes TD, Paul DR (2002) Polymer 43:6727

10. Tucker 1II CL, Liang CL (1999) Comp Sci Technol 59:655

11. Mori T, Tanaka K (1973) Acta Meiall 21:571

12. Benveniste Y (1987) Mech Mater 6:147

13. Draya D. Gilorminia P, Régnier G (2007) Comp Sci and Technol 67:1601

14. He J, Zoller $P(1994)$ J Pol So Par B Poi Fhys $32: 1049$

15. Perman, EP. Urry WD (1927) Proc mys Soc 40:186

16. Assouline E, Wachtel E, Grigtil S, Lustiger A, Wagner HD, Marcm G (2002) Macrom $35 ; 403$ 